Scleral buckle

\section{Is it time to call time on the scleral buckle?}

\section{McLeod}

\section{One can't help feeling that we are well past the beginning of the end}

O vernight, an extensive pool of subretinal fluid (SRF) disappears following the judicious application of a small plomb over a peripheral U-tear, and one can only marvel at the underlying mechanisms at play. The scleral indentation has somehow overcome the dynamic tractional forces operating in the region of the break, allowing it to close; and the "pigment epithelial pump" has obligingly dried out the subretinal space despite our having decimated the outer retinal tissues around the break in order to induce break sealing. Once the chorioretinal adhesion is fully established, it usually ensures that the break remains closed and the retina reattached even if the indentation eventually recedes and vitreoretinal traction is reinstated.

The attractions of the "Custodis" procedure are plain to see, not least because the complications of SRF drainage (and other scleral transgressions) are potentially avoided. ${ }^{12}$ However, the majority of rhegmatogenous retinal detachments (RRDs) cannot be treated so simply and effectively using a segmental buckle, especially where the breaks are too many or too large to indent with any degree of subtlety, too small or too well hidden to be identified with certainty, too awkward in location to be easily reached, or subject to too much traction. Twenty years ago, these challenges could be met by one or more of a series of surgical adjuncts, including encirclement of the globe (to relieve anterior circumferential traction and to ensure the maintenance of the indentations created by segmental buckles) ${ }^{1-3}$; intravitreal injection of air or gases of low solubility (to temporarily sequester the breaks from fluid vitreous that might otherwise be recruited subretinally $)^{45}$; or closed microsurgery combined with break tamponade (to eliminate tractional elements and opacities by vitrectomy while bringing air, gas, or silicone oil into direct relation with the breaks after hydraulic retinal reattachment). ${ }^{6-8}$ Which strategy a surgeon chose to pursue at that time depended more on the mesmeric influ- ence of the grand masters of vitreoretinal surgery than on the outcomes of formal clinical trials.' However, the benefits that vitrectomy brought to the management of particularly difficult detachments were so palpable that a randomised controlled trial could not have been ethically justified. ${ }^{10}$

Escoffery and his colleagues were among the first to have the temerity to undertake closed microsurgery without scleral buckling for RRDs in which the responsible breaks were regarded as being otherwise eminently "buckleable." ${ }^{11}$ Their thesis-that "vitrectomy and gas" might eventually supersede buckling for most types of primary RRD-acknowledged the fact that the more anteriorly a break is located, the more difficult it is for traction to be relieved internally and for SRF to be evacuated via the break. This is the converse of non-drainage buckling where anterior breaks settle most readily onto a scleral indentation. ${ }^{12}$ Nevertheless, the first hesitant steps had been taken towards internal surgery without external buckling becoming the procedure of choice for most primary RRDs.

Variations on the surgical theme at about the same time as Escoffery's publication were another reason for the faltering start to this evolutionary progression. In the United States, "pneumatic retinopexy" was popularised by Hilton and Grizzard. ${ }^{12}$ This depended on the capability of a relatively small intravitreal gas bubble to close breaks in the absence of scleral indentation, and it sometimes succeeded in cooperative patients with superior breaks despite the several inherent threats in using expanding gases in non-vitrectomised eyes. These included the potential for residual gel to compromise internal break tamponade, for continuing traction to militate against effective break sealing, and especially for new breaks to form. ${ }^{9}{ }^{13}$ In the United Kingdom meanwhile, the DACE surgical sequence (that is, Drainage of SRF followed by Air injection, Cryopexy around the reattached breaks and placement of a low profile Explant) was introduced for bullous RRDs. This order of surgical steps was designed to reduce the risk of haemorrhage during SRF drainage and to facilitate retinopexy and accurate break indentation. The results from two case series, one from St Thomas's Hospital and the other from Moorfields Eye Hospital, were published in the same issue of the BJO in $1985,1^{14}$ and the procedure enjoyed a significant following for some time on this side of the Atlantic.

Other developments then came along that were to reinvigorate the onward march of the vitrectomy and gas approach, not least the widespread adoption of phacoemulsification and wide angle fundus viewing. With the exception of some diabetic patients and patients under age $50,{ }^{16}$ vitrectomy invariably leads to the development of nuclear lens sclerosis (to which type of cataract eyes with RRD harbour a distinct susceptibility anyhow because of pre-existing vitreous syneresis). ${ }^{17}$ This represented a significant disadvantage until small incision phacosurgical techniques reached full maturity, ${ }^{9}$ but intraoperative or post-vitrectomy lens removal can now be carried out routinely with minimal risk of compromising the posterior segment surgery. Similarly, although internal searching for retinal breaks had been shown to be very effective (especially when combined with deep kinetic scleral indentation), ${ }^{18}$ new systems for wide angle viewing not only improved fundus visualisation and identification of peripheral retinal breaks (even through a small pupil), but their optics also made internal drainage of SRF and fluid/gas exchange in phakic eyes that much more straightforward. ${ }^{19}$ The use of heavy liquids to reattach the retina intraoperatively by squeezing SRF back out through the breaks, ${ }^{20}$ and close "shaving" of vitreous from the retina, ${ }^{21}$ have added to the sense of control over intraoperative events for the surgeon and to a perceived reduction in his/her need to employ additional external means of combating traction.

Once segmental buckles to indent vitrectomised breaks in the superior fundus had been abandoned, routine encirclement of the globe quickly fell from favour as well. Traditionally, encirclement as an adjunct to vitrectomy had been credited with counteracting residual vitreoretinal traction and with closing unseen breaks in the vitreous base region. These presumed attributes weren't subjected to any formal study, however, and in many centres encirclement was discarded without so much as a murmur. In others, several rows of 360 degree laser retinopexy replaced 
encirclement in fulfilling the surgeon's desire to tackle problems suspected to be lurking peripherally as, for example, in pseudophakic eyes. ${ }^{22}$ The encircling band having been dispatched to oblivion, this trend has latterly extended "full circle" to the recommended withdrawal of external buckling even for detachments from inferior tears (that is, those located between 4 o'clock clockwise to 8 o'clock near the equator or more anteriorly). ${ }^{23}$ These RRDs were formerly thought to require scleral indentation in order to achieve secure closure and sealing of the tears in the context of disadvantageous posturing and the risk of future break reopening from proliferative vitreoretinopathy (PVR), a process that particularly afflicts the inferior retina.

There is already justification for recommending avoidance of a long, wide buckle during primary surgery, with its attendant risk of vortex vein compression

As with the D-ACE sequence, independent case series from St Thomas's and Moorfields hospitals are published simultaneously in this issue of the BJO (pp 1372 and 1376) to provide mutual corroboration of the feasibility of this latest act of vitreoretinal surgical omission. Eighty one per cent and 89\% respectively of previously unoperated detachments with inferior tears were reattached at the first attempt using vitrectomy and gas without buckling. These success rates are similar to those reported for mixed primary detachments, whether buckled or not, in a national audit. ${ }^{24}$ Apparently, PVR wasn't a major problem in the Moorfields study, but the extent to which its malign influence caused or complicated the failure of break closure in one fifth of the St Thomas's cases, and then compromised the outcome of secondary surgery, isn't revealed in the bald statistics presented.

To further strengthen their argument, each group of authors wrapped up their case series in a retrospective study, the flaws in which are acknowledged. The St Thomas's group compared the outcomes of vitrectomy and gas for RRDs with inferior breaks with those for RRDs without inferior breaks that had been matched for patient age and sex (Wickham et al, this issue), though neither of these are important confounding variables in determining the results of RRD management. The Moorfields study compared treatment outcomes for RRDs with inferior breaks that had either been buckled or were non-buckled (Sharma et al, this issue) though the decision to buckle would inevitably have been biased by (more or less) tangible manifestations of early PVR. However, both centres emphasised the need to eliminate tractional elements from the vicinity of the breaks and for them to have achieved this objective with so little instrumental lens trauma is remarkable. The implication to be drawn is that excessively demanding postoperative posturing regimes need no longer be regarded as crucial to the successful management of unbuckled inferior breaks provided traction is thus minimised. Another factor may be the "enforced" reattachment of the retina and the use of a large gas bubble, measures that are not so critical in other circumstances. ${ }^{18}$

Does this mean, therefore, that the scleral buckle is now effectively dead and buried so far as the treatment of fresh RRDs from tractional breaks is concerned? Possibly. Further guidance may be forthcoming as and when a randomised trial of "segmental buckling versus vitrectomy and gas" reports. ${ }^{25}$ However, encirclement is permitted in both arms of this particular study, and it remains to be seen whether and how associated quality of life and economic issues will be addressed. For example, is the burden of posturing comparable to the ciliary neuralgia from ocular indentation in the immediate postoperative period, and how will fiscal matters surrounding the relative costs of consumables and disposables, and the prohibition of air travel, be analysed?

While we await this report, however, the possibility exists that the next generation of vitreoretinal surgeons, weaned on the sutureless incisions and fluidics of phacoemulsification, will be slower to acquire the necessary skills in external buckling anyway. Therefore, should the anatomical outcomes prove to be similar (as is likely), the balance of judgment will increasingly favour a procedure that expressly addresses vitreoretinal traction and vitreous opacities, and whose principal complication (namely, cataract) is eminently treatable, against one that doesn't and that has a galaxy of potential drawbacks whether refractive, oculomotor, circulatory, or directly explant related. ${ }^{9}$ The possibility that vital staining of the retina might improve intraoperative break detection in future, ${ }^{26}$ and emerging evidence that reattachment of the foveal retina after vitrectomy is more definitive than that following scleral buckling, ${ }^{27}$ can only add to the appeal of the internal approach. Indeed, given these latest reports from Moorfields and St Thomas's, there is already justification for recommending avoidance of a long, wide buckle during primary surgery for, say, an RRD from a tractional tear posterior to an inferior equatorial lattice patch, with its attendant risk of vortex vein compression.

What about the management of detachments resulting from multiple, inferiorly located, atrophic retinal holes and those caused by large oral dialyses? For the present, vitrectomy remains contraindicated for RRDs with no posterior vitreous detachment, but it shouldn't be too long before the vitreous gel can be safely separated intraoperatively from detached retina, using a biochemically targeted intervention, as a prelude to complete vitrectomy and gas tamponade. ${ }^{28}$ Notwithstanding the continuing allure of the non-drainage approach for single, small, anteriorly located breaks, therefore, one can't help feeling that we are well past the beginning of the end for the scleral buckle, particularly in primary RRDs uncomplicated by PVR.

Br J Ophthalmol 2004;88:1357-1359. doi: 10.1136/bjo.2004.050146

Correspondence to: D McLeod, Academic Department of Ophthalmology, Manchester Royal Eye Hospital, Oxford Road, Manchester M13 9WH, UK; david.mcleod@man.ac.uk

\section{REFERENCES}

1 Lincoff H, Baras I, McLean J. Modifications to the Custodis procedure for retinal detachment. Arch Ophthalmol 1965;73:160-3.

2 Michels RG. Scleral buckling methods for rhegmatogenous retinal detachment. Retina 1986;6: 1-49.

3 Schepens CL, Okamura ID, Brockhurst RJ, et al. Scleral buckling procedures. V. Synthetic sutures and silicone implants. Arch Ophthalmol 1960;64:868-81.

4 Rosengren B. Results of treatment of detachments of the retina with diathermy and injection of air into the vitreous. Acta Ophthalmol 1938; 16:573-9

5 Norton EWD. Intraocular gas in the management of selected retinal detachments. Trans Am Acad Ophthalmol Otolaryngol 1973;77:OP85-98.

6 Machemer R, Buettner H, Norton EWD, et al. Vitrectomy. A pars plana approach. Trans Am Acad Ophthalmol Otolaryngol 1971;75:813-20.

7 Charles S. Vitreous microsurgery. Baltimore: Williams and Wilkins, 1981.

8 Leaver PK, Cooling RJ, Feretis EB, et al. Vitrectomy and fluid/silicone-oil exchange for giant retinal tears: results at six months. Br J Ophthalmol 1984;68:432-8.

9 Wilkinson CP. What is the 'best' way to fix a routine retinal detachment? Chapter 9, pp 85102. In: Lewis H, Ryan SJ, eds. Medical and surgical retina. St Louis: Mosby, 1994.

10 Freedman B. Equipoise and the ethics of clinical research. N Engl J Med 1987:317:141-5.

11 Escoffery RF, Olk RJ, Grand MG, et al. Vitrectomy without scleral buckling for primary rhegmatogenous retinal detachment Am J Ophthalmol 1985;99:275-81.

12 Hilton GF, Grizzard WS. Pneumatic retinopexy A two-step outpatient operation without conjunctival incision. Ophthalmology 1986;93:626-41.

13 Grizzard WS, Hilton GF, Hammer ME, et al. Pneumatic retinopexy failures. Cause, prevention, timing, and management. Ophthalmology 1995; 102:929-36.

14 Stanford MR, Chignell AH. Surgical treatment of superior bullous rhegmatogenous retinal detachments. Br J Ophthalmol 1985;69:729-32. 
15 Gilbert C, McLeod D. D-ACE surgical sequence for selected bullous retinal detachments. Br J Ophthalmol 1985;69:733-6.

16 Thompson JT. The role of patient age and intraocular gas use in cataract progression after vitrectomy for macular holes and epiretinal membranes. Am J Ophthalmol 2004; 137:250-7.

17 Harocopos GJ, Shui Y-B, McKinnon M et al. Importance of vitreous liquefaction in age-related cataract. Invest Ophthalmol Vis Sci 2004;45:77-85.

18 Rosen PH, Wong HC, McLeod D. Indentation microsurgery: internal searching for retinal breaks. Eye 1989:3:277-81.

19 Spitznas M. A binocular indirect ophthalmomicroscope (BIOM) for non-contact wide-angle vitreous surgery. Graefes Arch Clin Exp Ophthalmol 1987;225:13-5.
20 Chang S. Low viscosity liquid fluorochemicals in vitreous surgery. Am J Ophthalmol 1987; 103:38-43.

21 Oyagi T, Emi K. Vitrectomy without scleral buckling for proliferative vitreoretinopathy. Retina 2004;24:215-18.

22 Campo RV, Sipperley JO, Sneed SR, et al. Pars plana vitrectomy without scleral buckle for pseudophakic retinal detachments. Ophthalmology 1999;106:1811-16.

23 Tanner V, Minihan M, Williamson TH. Management of inferior breaks during pars plana vitrectomy for retinal detachment. $\mathrm{Br} J$ Ophthalmol 2001:85:480-2.

24 Thompson JA, Snead MP, Billington BM, et al. National audit of the outcome of primary surgery for rhegmatogenous retinal detachment. II. Clinical outcomes. Eye 2002;16:771-7.
25 Heimann H, Hellmich M, Bornfeld N, et al. Scleral buckling versus primary vitrectomy in rhegmatogenous retinal detachment (SPR Study): design issues and implications. SPR Study report no 1. Graefes Arch Clin Exp Ophthalmo 2001;239:567-74.

26 Jackson TL, Marshall J. Fluorophore-assisted retinal break detection using antibodies to glia fibrillary acidic protein. Invest Ophthalmol Vis Sci 2004;45:993-1001.

27 Wolfensberger TJ. Foveal reattachment after macula-off retinal detachment occurs faster after vitrectomy than after buckle surgery. Ophthalmology 2004;111:1340-3.

28 Ramesh S, Bonshek RE, Bishop PN Immunolocalisation of opticin in the human eye. Br J Ophthalmol 2004;88:697-702.

\section{Orbital steroid injections}

\section{R A Goldberg}

\section{The ugly sister}

n this issue of the BJO (p 1380), Ebner and colleagues present data from their pilot study of orbital steroids in Graves' orbitopathy. Even though the patient population is small, and the treatment groups not entirely comparable, the study does provide some evidence that steroid injections used to treat Graves' orbitopathy may be safe and efficacious.

Orbital steroid injections for Graves' orbitopathy and other inflammatory disease of the orbit have had a somewhat chequered past. During my training under Norman Shorr, MD, at the Jules Stein Eye Institute, UCLA, I observed frequent use of orbital steroid injections to treat orbital inflammatory disease including Graves, and I also learned about steroid injections from William Stewart, MD, in San Francisco. As I subsequently travelled to different institutions as a visiting speaker, I was surprised therefore to find that in many programmes orbital steroids were never used, either because of concerns about safety or concerns about efficacy. Compared to oral steroids, they were considered the ugly sister.

Certainly, corticosteroids are a potentially dangerous medicine with a long track record of proved ability to cause significant complications. In addition to the systemic complications of steroids, injection around the eye also poses the risk of local complications including globe perforation, ${ }^{1-3}$ intractable elevated intraocular pressure, ${ }^{45}$ conjunctival or corneoscleral melting, ${ }^{6-8}$ vascular occlusion from embolisation or pressure induced optic nerve compression, ${ }^{9-16}$ proptosis or fat atrophy, ${ }^{17-19}$ depigmentation, and granuloma related to the methyl cellulose vehicle of the depot injection. However, I suggest that reluctance to use orbital steroid injections derives its legacy from complications of retrobulbar steroid injections used to treat intraocular processes. Retrobulbar or sub-Tenon's injections are given with a long needle, and the medication is very close to or in direct contact with the globe.

The technique for orbital injections is substantially different from retrobulbar injection. For orbital injections, the medication is placed just inside the septum near the orbital rim. A half inch needle is used (I prefer a 27 gauge

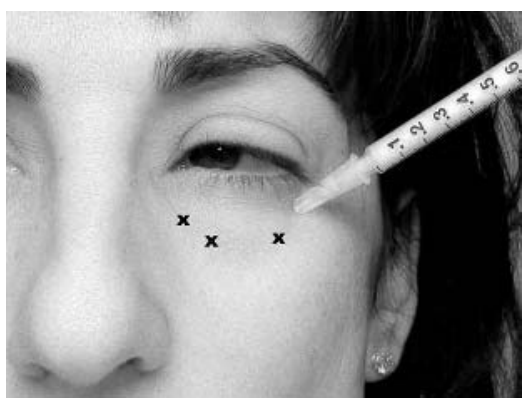

Figure 1 Triamcinolone acetonide $40 \mathrm{mg} / \mathrm{ml}$, $0.8-1 \mathrm{ml}$, is injected just inside the orbital rim, posterior to the orbital septum. The injection is given slowly using a half inch 27 gauge needle. " $X$ " indicates other common sites; the medial, lateral, and superior orbit are technically more difficult and used less often. needle to allow more easy passage of the particulate injection) and the risk of perforating the globe is remote. Also, the small short needle is well tolerated by patients: by using distraction techniques and a gentle touch, injections can be given almost painlessly. Perhaps because the medication is not in contact with the globe, severe elevation of intraocular pressure is not typically seen (although I would be careful in recommending orbital steroid injections in patients with significant glaucoma). Triamcinolone acetonide (Kenalog) is manufactured in a $40 \mathrm{mg} / \mathrm{ml}$ concentration, so no more than $1 \mathrm{ml}$ of fluid is needed. Particularly in the anterior orbital location, with slow injection, I have not observed and would not expect a $1 \mathrm{ml}$ injection to create a vision threatening increase in orbital pressure. Slow injection using the small gauge needle is also important in order to minimise the risk of intravascular injection forcing retrograde flow of particulate matter into the retinal circulation. ${ }^{12} 20$

Efficacy is a difficult thing to measure in Graves' orbitopathy; the heterogeneity of the disease and the tendency for spontaneous improvement, create a requirement for carefully controlled studies with adequate numbers of patients in order to assess and compare treatment options. I am not aware of any studies of steroid injections, including the pilot study published here by Ebner et al, that definitively answer the question of efficacy of steroid injections relative to disease outcome. ${ }^{21}$

On the other hand, no clinician who cares for patients with Graves' orbitopathy would likely dispute the efficacy of corticosteroids in ameliorating the acute inflammatory features of the disease. Corticosteroids have been a mainstay of treatment, and often provide rapid improvement in symptoms and in clinical evidence of inflammation. Whether the temporary improvement observed with corticosteroid therapy affects the final outcome of 
the disease is a fascinating and unanswered question, but at least the temporary improvement offered by corticosteroids often allows these patients to resume daily activities.

We should not require studies of injected steroids to show proof that they fundamentally change the disease. The pertinent question is whether steroid injections can achieve the improvement in symptoms that we observe with oral corticosteroids, without an unacceptable rate of local complications, and with decreased systemic side effects. The anecdotal experience of those who use this delivery mechanism suggests that injected steroids do provide symptom relief. Although there is some systemic absorption, ${ }^{21}{ }^{22}$ pharmacological studies of intra-articular injections suggest good compartmentalisation ${ }^{22}$ and anecdotal experience, as well as Ebner's study, suggest decreased systemic side effects compared to oral steroids. With attention to careful technique, I believe they can be safely used (although patients have to accept the rare risks of steroid injections which include blindness). In the absence of large studies to accurately determine the true complication rate, practitioners should be encouraged to report major complications to the medical community.

Important technical points to improve the safety of injection include the use of a short half inch 27 gauge needle, slow injection, injection site in the anterior orbit just posterior to the orbital septum (fig 1), and limitation of injected volume to $1 \mathrm{ml}$ or less. The depot injection lasts for about 30 days (without prompting, patients often volunteer that the improvement in symptoms wears off after a month) so that I personally use a dosing schedule of one injection per month, repeated as needed. I have injected as many as 13 aliquots in patients with recurring orbital myositis, but on average, I find that patients with Graves' orbitopathy need treatment for 3-6 months. My anecdotal experience is that a $40 \mathrm{mg}$ injection provides an effect equivalent to $20 \mathrm{mg}$ daily of oral prednisone, so that patients who can be controlled at that oral dose are good candidates. Systemic side effects are minimal, or at least reduced, compared with oral steroids. I am sure that there is some placebo effect associated with the dramatic experience of an injection next to the eye, which works in our favour as well.

Our patients with Graves' orbitopathy have a tough battle. They have a frustrating disease that still awaits a cure. The more options that we have for medical and surgical symptomatic treatment, the better we can help them through the stages of their disease, and get them back to a comfortable, productive life. Orbital steroid injections seem to deliver a moderate dose of depot corticosteroid to the orbit with an acceptable local complication rate and with decreased systemic toxicity relative to oral corticosteroids.

\section{ACKNOWLEDGEMENTS}

Support provided by the Dr Bernard and Jenny Nelson Family Trust.

I thank Michael Roberts, MD, and Guy Ben Simon, MD, who provided valuable assistance in the preparation of this manuscript.

Br J Ophthalmol 2004;88:1359-1360.

doi: 10.1136/bjo.2004.051755

Correspondence to: R A Goldberg, Orbital Disease Center, Jules Stein Eye Institute, David Geffen School of Medicine at UCLA, 100 Stein Plaza, Los Angeles, CA 90095 USA; goldberg@jsei.ucla.edu

\section{REFERENCES}

1 Giles CL. Bulbar perforation during periocular injection of corticosteroids. Am J Ophthalmol 1974:77:438-41.

2 Schlaegel TF, Wilson FM. Accidental intraocular injection of depot corticosteroids. Trans Am Acad Ophthalmol Otolaryngol 1974;78:847-55.

3 Hosal BM, Zilelioglu G. Ocular complication of intralesional corticosteroid injection of a chalazion. Eur J Ophthalmol 2003;13:798-9.

4 Herschler J. Intractable intraocular hypertension induced by repository triamcinolone acetonide. Am J Ophthalmol 1972;74:501-4.
5 Akduman L, Kolker AE, Black DL, et al. Treatment of persistent glaucoma secondary to periocular corticosteroids. Am J Ophthalmol 1996:122:275-7.

6 Fogla R, Rao SK, Biswas J. Avoiding conjunctival necrosis after periocular depot corticosteroid injection. J Cataract Refractive Surg 2000;26:163-4

7 Agrawal S, Agrawal J, Agrawal TP. Conjunctival ulceration following triamcinolone injection. Am J Ophthalmol 2003;136:539-40.

8 Jabs DA. A prospective evaluation of subconjunctival injection of triamcinolone acetonide for resistant anterior scleritis. (Discussion by Ds A Jabs) Ophthalmology, 2002;109:806-7.

9 Ellis PP. Occlusion of the central retinal artery after retrobulbar corticosteroid injection. Am J Ophthalmol 1978:85:352-6.

10 Moshfeghi DM, Lowder CY, Roth DB, Kaiser PK Retinal and choroidal vascular occlusion after posterior sub-tenon triamcinolone injection. Am J Ophthalmol 2002;134:132-4.

11 Thomas EL, Laborde RP. Retinal and choroidal vascular occlusion following intralesional corticosteroid injection of a chalazion. Ophthalmology 1986:93:405-7.

12 Shorr N, Seiff SR. Central retinal artery occlusion associated with periocular corticosteroid injection for juvenile hemangioma. Ophthalmic Surg 1986; 17:229-31.

13 McLean EB. Inadvertent injection of corticosteroid into the choroidal vasculature. Am J Ophthalmol 1975:80:835-7.

14 Mabry RL. Visual loss after intranasal corticosteroid injection. Arch Otolaryngol 1981;107:484-6.

15 McCleve DE, Goldstein JC. Blindness secondary to injections in the nose, mouth and face: cause and prevention. Ear, Nose Throat J 1995;74:182-8.

16 McGrew RN, Wilson RS, Havener WH. Sudden blindness secondary to injections of common drugs in the head and neck: clinical experiences. Otolaryngology 1978;86:147-51.

17 Gupta OP, Boynton JR, Sabini P, et al. Proptosis after retrobulbar corticosteroid injections. Ophthalmology 2003;110:443-7.

18 Smith JR, George RK, Rosenbaum JT. Lower eyelid herniation of orbital fat may complicate periocular corticosteroid injection. Am J Ophthalmol 2002;133:845-7.

19 Nozik RA. Orbital rim fat atrophy after repository periocular corticosteroid injection. Am J Ophthalmol 1976;82:928-30.

20 Egbert JE, Paul S, Engel WK, et al. High injection pressure during intralesional injection of corticosteroids into capillary hemangiomas. Arch Ophthalmol 2001;119:677-83.

21 Goyal R, Watts P, Lane CM, et al. Adrenal suppression and failure to thrive after steroid injections for periocular hemangioma. Ophthalmology 2004;111:389-95.

22 Derendorf H, Mollmann H, Gruner A, et al. Pharmacokinetics and pharmacodynamics of glucocorticoid suspensions after intra-articular administration. Clin Pharmacol Ther 1986;39:313-17. 